\title{
The distribution of $\mathrm{ND}_{2} \mathrm{H}$ in LDN $1689 \mathrm{~N}$
}

\author{
M. Gerin ${ }^{1}$, D. C. Lis ${ }^{2}$, S. Philipp ${ }^{3}$, R. Güsten ${ }^{3}$, E. Roueff ${ }^{4}$, and V. Reveret ${ }^{5}$ \\ 1 LERMA, CNRS UMR 8112, Observatoire de Paris and ENS, 24 rue Lhomond, 75231 Paris Cedex 05, France \\ e-mail: gerin@lra.ens. fr \\ 2 California Institute of Technology, MC 320-47, Pasadena, CA 91125, USA \\ e-mail: dcl@submm.caltech.edu \\ 3 Max-Planck-Institut für Radioastronomie, Auf dem Hügel 69, Bonn, Germany \\ e-mail: philipp,guesten@mpifr-bonn.mpg.de \\ 4 LUTH, CNRS UMR 8102, Observatoire de Paris and Université Paris 7, Place J. Janssen, 92190 Meudon, France \\ e-mail: evelyne.roueff@obspm.fr \\ 5 European Southern Observatory, Casilla 19001, Santiago 19, Chile \\ e-mail: vreveret@eso.org
}

Received 10 April 2006 / Accepted 1 June 2006

\section{ABSTRACT}

\begin{abstract}
Aims. Finding tracers of the innermost regions of prestellar cores is important for understanding their chemical and dynamical evolution before the onset of gravitational collapse. While classical molecular tracers, such as CO and CS, have been shown to be strongly depleted in cold, dense gas by condensation on grain mantles, it has been a subject of discussion to what extent nitrogen-bearing species, such as ammonia, are affected by this process. As deuterium fractionation is efficient in cold, dense gas, deuterated species are excellent tracers of prestellar cores. A comparison of the spatial distribution of neutral and ionized deuterated species with the dust continuum emission can thus provide important insights into the physical and chemical structure of such regions.

Methods. We study the spatial distribution of the ground-state $335.5 \mathrm{GHz}$ line of $\mathrm{ND}_{2} \mathrm{H}$ in LDN 1689N, using APEX, and compare it with the distribution of the $\mathrm{DCO}^{+}(3-2)$ line, as well as the $350 \mu \mathrm{m}$ dust continuum emission observed with the SHARC II bolometer camera at CSO.

Results. While the distribution of the $\mathrm{ND}_{2} \mathrm{H}$ emission in $\mathrm{LDN} 1689 \mathrm{~N}$ is generally similar to that of the $350 \mu \mathrm{m}$ dust continuum emission, the peak of the $\mathrm{ND}_{2} \mathrm{H}$ emission is offset by $\sim 10^{\prime \prime}$ to the East from the dust continuum and $\mathrm{DCO}^{+}$emission peak. $\mathrm{ND}_{2} \mathrm{H}_{\text {and }} \mathrm{ND}_{3}$ share the same spatial distribution. The observed offset between the $\mathrm{ND}_{2} \mathrm{H}$ and $\mathrm{DCO}^{+}$emission is consistent with the hypothesis that the deuterium peak in LDN $1689 \mathrm{~N}$ is an interaction region between the outflow shock from IRAS 16293-2422 and the dense ambient gas. We detect the $J=4 \rightarrow 3$ line of $\mathrm{H}^{13} \mathrm{CO}^{+}$at $346.998 \mathrm{GHz}$ in the image side band serendipitously. This line shows the same spatial distribution as $\mathrm{DCO}^{+}(3-2)$, and peaks close to the $350 \mu \mathrm{m}$ emission maximum which provides further support for the shock interaction scenario.
\end{abstract}

Key words. ISM: molecules - ISM: individual objects: LDN 1689N - ISM: clouds - radio lines: ISM - submillimeter

\section{Introduction}

Deuteration of nitrogen compounds, such as ammonia and $\mathrm{N}_{2} \mathrm{H}^{+}$, is spectacular in a number of environments including dark clouds, such as L134N (Tiné et al. 2000; Roueff et al. 2000; Roueff et al. 2005), low-mass star forming regions and prestellar cores such as LDN 1689N and Barnard 1 (Loinard et al. 2001; Gerin et al. 2001; Lis et al. 2002a). The discussion of the respective contributions of grain and gas-phase processes in the deuteration is active, but no definite solution is yet available. The lack of detection of deuterated water in ices toward low-mass young stellar objects (YSOs) by Dartois et al. (2003) and Parise et al. (2003) suggests that "another mechanism than pure solid state chemistry may be active to produce very high deuterium enrichment in the gas phase".

Further constraints on the deuterium fractionation mechanisms are provided by the spatial distribution of deuterated species, as compared with the molecular gas distribution traced by the submillimeter dust continuum emission. Whereas maps of singly deuterated species have been published in many cores
- the relatively large line intensities of e.g. $\mathrm{DCO}^{+}, \mathrm{N}_{2} \mathrm{D}^{+}$allow easy mapping with state of the art detectors - mapping multiply deuterated species has proven to be a challenge, given the relatively low line intensities. Ceccarelli et al. (2001) have shown that the $\mathrm{D}_{2} \mathrm{CO}$ emission is extended around the class 0 protostar IRAS 16293-2422. Roueff et al. (2005) present a limited map of the $\mathrm{ND}_{3}$ ground-state transition at $309 \mathrm{GHz}$ with the $\mathrm{CSO}$ in LDN $1689 \mathrm{~N}$, which unfortunately suffers from rather poor pointing accuracy. Because the ground state $\mathrm{ND}_{2} \mathrm{H} 1_{0,1}-0_{0,0}$ lines, at 335.5 for the ortho species and $335.4 \mathrm{GHz}$ for the para species (See Coudert \& Roueff (2006) for $\mathrm{NH}_{3}$ and its isotopologues line frequencies), are relatively strong $(0.6 \mathrm{~K}$ in LDN $1689 \mathrm{~N}$; Lis et al. 2006) and the atmospheric transmission is good at this frequency, we have carried out the first map of a doubly deuterated species in a dense core.

\section{Observations}

The observations have been carried out using the APEX-2a $350 \mathrm{GHz}$ receiver of the Atacama Pathfinder Experiment 

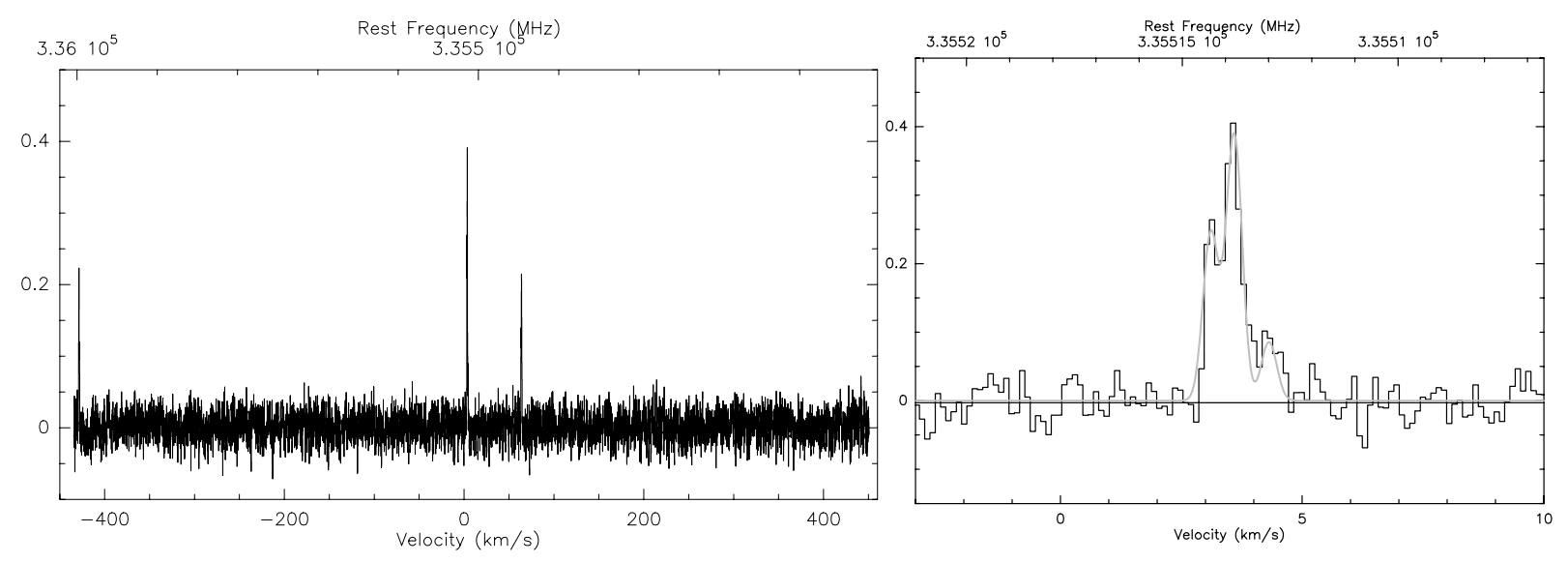

Fig. 1. Left: full spectrum averaged over the map. p- $\mathrm{ND}_{2} \mathrm{H}\left(1_{11}-0_{00}\right)$ at $335.446 \mathrm{GHz}$, o- $\mathrm{ND}_{2} \mathrm{H}\left(1_{11}-0_{00}\right)$ at $335.514 \mathrm{GHz}$ and $\mathrm{H}^{13} \mathrm{CO}^{+}(4-3)$ at $346.998 \mathrm{GHz}$ (in the image side band) are detected. Right: enlargement showing a fit of the hyperfine structure of the o- $\mathrm{ND}_{2} \mathrm{H}$ line. The temperature scale is $T_{\mathrm{mb}}(\mathrm{K})$ and the velocity scale is relative to the LSR.

$\left(\mathrm{APEX}^{1}\right)$. We combined data obtained during two periods, August 2005 and October 2005. The receiver was tuned in DSB with the $\mathrm{ND}_{2} \mathrm{H}$ lines in the lower sideband. The backend was the facility MPIfR Fast Fourier Spectrometer. We observed a total of 10 positions towards LDN $1689 \mathrm{~N}\left(\alpha_{2000}=16: 32: 29.470\right.$, $\left.\delta_{2000}=-24: 28: 52.60\right)$. The pointing was checked regularly and found accuracte to $\sim 2-3^{\prime \prime}$. The observations have been taken in the position-switched mode with a reference position located $240^{\prime \prime}$ East of the source for the August 2005 data. The October 2005 data have been taken with a reference position offset by $10^{\prime}$ in azimuth. Line and continuum maps shown in Lis et al. (2002b) and Stark et al. (2004) show that the source emission rapidly drops towards the East.

The data have been corrected for the sideband gain and the APEX main beam efficiency of 0.7 at $335 \mathrm{GHz}$. The temperature scale used in this paper is the main beam brightness temperature scale. Overall, the data calibration agree well with the CSO spectrum presented by Lis et al. (2006). The FWHM beam size of APEX is $18^{\prime \prime}$ at $335.5 \mathrm{GHz}$.

\section{Results}

Figure 1 shows the average spectrum integrated over the map, while contour maps are shown in Fig. 2. The ortho- and para$\mathrm{ND}_{2} \mathrm{H}\left(1_{11}-0_{00}\right)$ lines are clearly detected with a mean intensity ratio of 2:1, reproducing the ortho-to-para statistical weight ratio (Lis et al. 2006). We have also detected the $\mathrm{H}^{13} \mathrm{CO}^{+}(4-3)$ line at $346.998 \mathrm{GHz}$ in the image side band, which is discussed below.

\section{1. $\mathrm{ND}_{2} \mathrm{H}$ spatial distribution}

Figure 2 shows contours of the main $\mathrm{ND}_{2} \mathrm{H}$ emission at $335.5 \mathrm{GHz}$ (in white) overlayed over a $350 \mu \mathrm{m}$ continuum image. The $\mathrm{ND}_{2} \mathrm{H}$ emission is clearly extended and shows an elongated shape generally similar to the dust continuum emission. The limited $\mathrm{ND}_{3}$ map shown by Roueff et al. (2005) shows the same elongated pattern. Over the limited extend of the $\mathrm{ND}_{2} \mathrm{H}$ map, the line and continuum emissions are positively

1 This publication is based on data acquired with the Atacama Pathfinder Experiment (APEX). APEX is a collaboration between the Max-Planck-Institut für Radioastronomie, the European Southern Observatory and the Onsala Space Observatory. correlated with a correlation coefficient of 0.29 . This rather weak correlation is due to the difference in peak positions of the $\mathrm{ND}_{2} \mathrm{H}$ line emission with respect to the continuum peak, the $\mathrm{ND}_{2} \mathrm{H}$ emission beeing offset by $\sim 10^{\prime \prime}$ to the East of the dust contimuum emission maximum. This offset far exceeds the pointing uncertainties $\left(\sim 2-3^{\prime \prime}\right)$.

The right panel in Fig. 2 shows an overlay of the $\mathrm{ND}_{2} \mathrm{H}$ contours on a $\mathrm{DCO}^{+}(3-2)$ map (Lis et al. 2002b). The $\mathrm{ND}_{2} \mathrm{H}$ peak is clearly offset from the $\mathrm{DCO}^{+}$peak too. $\mathrm{D}_{2} \mathrm{CO}$ (Ceccarelli et al. 2000), $\mathrm{ND}_{3}$ (Roueff et al. 2005), $\mathrm{ND}_{2} \mathrm{H}$ (Loinard et al. 2001; Roueff et al. 2005), $\mathrm{DCO}^{+}$(Lis et al. 2002b), all appear at a blueshifted velocity compared to the cloud envelope: $V_{\mathrm{LSR}}=$ $3.4-3.6 \mathrm{~km} \mathrm{~s}^{-1}$ versus $V_{\mathrm{LSR}}=3.8-4.0 \mathrm{~km} \mathrm{~s}^{-1}$ for $\mathrm{C}^{18} \mathrm{O}$ and $\mathrm{C}^{17} \mathrm{O}$ (Stark et al. 2004). This velocity difference was first recognized by Lis et al. (2002b) who suggested that it results from the shock interaction of the powerful blue lobe of the IRAS 16293-2422 outflow with the dense core. The blue lobe of the molecular outflow is interacting and compressing LDN $1689 \mathrm{~N}$, creating dense and cold post-shock gas, blueshifted relative to the cloud envelope. The deuterated species preferably sample this cold and dense material as deuterium fractionation is more efficient at low temperatures and high gas densities. The large-scale $\mathrm{CO}$ and continuum maps presented by Lis et al. (2002b) and Stark et al. (2004) present convincing evidence for this scenario.

The spatial offset between $\mathrm{DCO}^{+}$and $\mathrm{ND}_{2} \mathrm{H}$ provides additional support for the $\mathrm{C}$-shock hypothesis. The $\mathrm{DCO}^{+}$ion is detected ahead of the neutral species $\mathrm{ND}_{2} \mathrm{H}$, closer to IRAS 16293-2422. The spatial separation, $10^{\prime \prime}$, or $\sim 0.01 \mathrm{pc}$, is consistent with $\mathrm{C}$-shock models with a pre-shock density of $10^{4} \mathrm{~cm}^{-3}$ and a magnetic field of $30 \mu \mathrm{G}$ (Lesaffre et al. 2004), which predict a total shock size of $0.04 \mathrm{pc}$. The deuterium chemistry in shocks has been previously studied by Pineau des Forêts et al. (1989) and Bergin et al. (1999) in different contexts. Because of the sensitivity of deuterium fractionation to both the gas temperature and the molecular depletion, it is expected that $\mathrm{DCO}^{+}$will be reformed more rapidly than $\mathrm{ND}_{2} \mathrm{H}$ in the post-shock gas. While $\mathrm{DCO}^{+}$is formed as soon as the gas temperature drops, efficient $\mathrm{ND}_{2} \mathrm{H}$ formation requires both cold temperatures and significant $\mathrm{CO}$ depletions, which are expected to occur downstream (Bergin et al. 1999). A spatial offset could therefore exist between abundance peaks of $\mathrm{DCO}^{+}$and $\mathrm{ND}_{2} \mathrm{H}$, as we have detected. More detailed shock models, would be able to further test the validity of this scenario. 

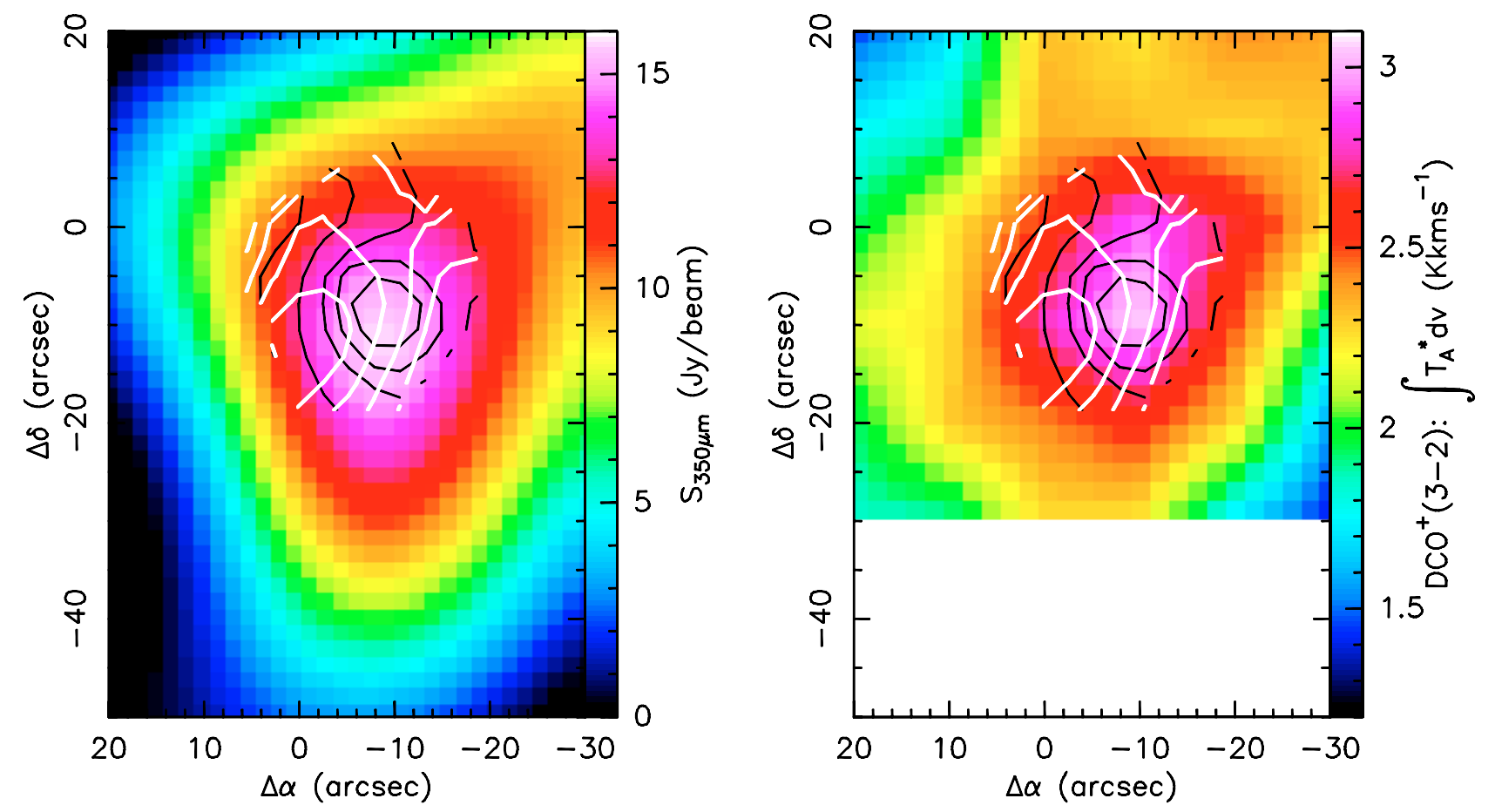

Fig. 2. Left: color image of the $350 \mu \mathrm{m}$ dust continuum emission obtained with SHARC II at the CSO, convolved to 20" angular resolution. The intensity scale is given in Jy/beam. White contours show the integrated intensity of the $\mathrm{o}-\mathrm{ND}_{2} \mathrm{H}$ line at $335.5 \mathrm{GHz}$, with contour levels from 0.25 to $0.45 \mathrm{~K} \mathrm{~km} \mathrm{~s}^{-1}$ with $0.05 \mathrm{~K} \mathrm{~km} \mathrm{~s}^{-1}$ spacing. Black contours show the integrated intensity of the $\mathrm{H}^{13} \mathrm{CO}^{+}(4-3)$ line at $346.998 \mathrm{GHz}$, with contour levels from 0.25 to $0.87 \mathrm{~K} \mathrm{~km} \mathrm{~s}^{-1}$ with $0.125 \mathrm{~K} \mathrm{~km} \mathrm{~s}^{-1}$ spacing. The map center is $\alpha_{2000}=16: 32: 29.470, \delta_{2000}=-24: 28: 52.60$. Right: the same contours plotted on a $\mathrm{DCO}^{+}(3-2)$ map (Lis et al. 2002b).

Table 1.

\begin{tabular}{cccccc}
\hline \hline $\begin{array}{c}\text { Position } \\
(\operatorname{arcsec})\end{array}$ & $\begin{array}{c}V \\
\mathrm{~km} \mathrm{~s}^{-1}\end{array}$ & $\begin{array}{c}\delta V \\
\mathrm{~km} \mathrm{~s}^{-1}\end{array}$ & $\begin{array}{c}N\left(\mathrm{ND}_{2} \mathrm{H}\right)^{1} \\
10^{13} \mathrm{~cm}^{-2}\end{array}$ & $\begin{array}{c}N\left(\mathrm{ND}_{3}\right)^{2} \\
10^{12} \mathrm{~cm}^{-2}\end{array}$ & $\begin{array}{c}N\left(\mathrm{H}_{2}\right)^{3} \\
10^{22} \mathrm{~cm}^{-2}\end{array}$ \\
\hline$-10,10$ & $3.63 \pm 0.02$ & $0.33 \pm 0.03$ & $9.8 \pm 2$ & & $4.1 \pm .5$ \\
10,0 & $3.53 \pm 0.02$ & $0.20 \pm 0.07$ & $3.6 \pm 1$ & & $3.1 \pm .5$ \\
0,0 & $3.56 \pm 0.02$ & $0.40 \pm 0.05$ & $12.7 \pm 2$ & 1.5 & $5.1 \pm .5$ \\
$-10,0$ & $3.62 \pm 0.02$ & $0.39 \pm 0.05$ & $10.0 \pm 2$ & & $5.9 \pm .5$ \\
$-20,0$ & $3.75 \pm 0.1$ & $0.8 \pm 0.3$ & $8.9 \pm 2$ & & $5.4 \pm .5$ \\
$0,-10$ & $3.60 \pm 0.02$ & $0.30 \pm 0.05$ & $15.8 \pm 2$ & & $5.8 \pm .5$ \\
$-10,-10$ & $3.70 \pm 0.05$ & $0.55 \pm 0.1$ & $11.7 \pm 2$ & 1.8 & $7.1 \pm .5$ \\
$-20,-10$ & $3.55 \pm 0.02$ & $0.26 \pm 0.05$ & $4.6 \pm 1$ & & $5.7 \pm .5$ \\
$0,-20$ & $3.69 \pm 0.02$ & $0.45 \pm 0.06$ & $12.7 \pm 2$ & 2.6 & $5.2 \pm .5$ \\
$-10,-20$ & $3.72 \pm 0.03$ & $0.58 \pm 0.08$ & $8.9 \pm 2$ & & $6.5 \pm .5$ \\
\hline
\end{tabular}

${ }^{1}$ Derived assuming LTE and $T_{\mathrm{ex}}=5 \mathrm{~K} .{ }^{2}$ Roueff et al. (2005). ${ }^{3}$ Derived assuming $T_{\text {dust }}=16 \mathrm{~K}$ and $\kappa_{350}=0.07 \mathrm{~cm}^{2} \mathrm{~g}^{-1}$.

\section{2. $N D_{2} \mathrm{H}$ abundance}

We have fitted the $\mathrm{ND}_{2} \mathrm{H}$ spectra using the hyperfine fitting command in CLASS. Although the signal-to-noise ratio is not high enough for securely deriving the line opacity at all positions, it is clear that the maximum opacity coincides with the maximum signal. The opacity derived for the average of all positions excluding $\left(10^{\prime \prime}, 0^{\prime \prime}\right)$ is $0.2 \pm 0.48$, and the corresponding excitation temperature is $8 \pm 3 \mathrm{~K}$, in good agreement with Lis et al. (2006). The spectra show some indication of higher line opacity, and lower excitation temperature $\left(\tau=3.11 \pm 1.5 ; T_{\mathrm{ex}}=5 \pm 1\right.$ towards the two positions with highest intensities $\left(0,-10^{\prime \prime}\right)$ and $\left(0,-20^{\prime \prime}\right)$.

The $\mathrm{ND}_{2} \mathrm{H}$ column densities have been derived assuming optically thin emission, LTE and using a uniform excitation temperature of $5 \mathrm{~K}$. They are listed, together with the $\mathrm{H}_{2}$ column density derived from dust continuum measurements in
Table 1. We use the $350 \mu \mathrm{m}$ dust continuum map obtained with SHARC II at the CSO (Fig. 2), and a a dust opacity of $\kappa_{350}=0.07 \mathrm{~cm}^{2} \mathrm{~g}^{-1}$ corresponding to $\kappa_{1300}=0.005 \mathrm{~cm}^{2} \mathrm{~g}^{-1}$ (Ossenkopf \& Henning 1994) for a dust emissivity index $\beta=2$, and a dust temperature of $16 \mathrm{~K}$ (Stark et al. 2004). The $350 \mu \mathrm{m}$ continuum fluxes are in fair agreement with the values reported by Stark et al. (2004), but on the lower side: we detect a maximum intensity of $16 \mathrm{Jy}$ in a $20^{\prime \prime}$ beam, to be compared with $19.4 \pm 2.3$ Jy in Stark et al. (2004). This dicrepancy may be an indication that some low-level entended emission is filtered out in the SHARC II image, which has been obtained in the "AC-biased mode", without the secondary chopper. The resulting $\mathrm{H}_{2}$ column densities reported in Table 1 could therefore be affected by this $35 \%$ uncertainty.

The $\mathrm{H}_{2}$ column density ranges from $3.1 \times 10^{22} \mathrm{~cm}^{-2}$ to $7.2 \times 10^{22} \mathrm{~cm}^{-2}$ across the map. The corresponding $\mathrm{ND}_{2} \mathrm{H}$ abundances varies from $0.7 \times 10^{-9}$ to $2.6 \times 10^{-9}$ relative to $\mathrm{H}_{2}$, with a 
mean value of $1.8 \times 10^{-9}$, in good agreement with previous work (Lis et al. 2006). $\mathrm{ND}_{2} \mathrm{H}$ appears to be remarkably abundant in this dense core. For the three positions where reliable $\mathrm{ND}_{3}$ data exist (Roueff et al. 2005), the $\left[\mathrm{ND}_{3}\right] /\left[\mathrm{ND}_{2} \mathrm{H}\right]$ abundance ratio is $0.01-0.02$, and seems to increase with increasing $\mathrm{ND}_{2} \mathrm{H}$ column density.

\section{3. $\mathrm{H}^{13} \mathrm{CO}^{+}$}

A relatively strong line is detected at the lower end of the velocity range in Fig. 1. Careful tests have been performed using the CSO and APEX telescopes for identifying this line. It turns out that the line can be assigned to the $J=4 \rightarrow 3$ transition of $\mathrm{H}^{13} \mathrm{CO}^{+}$at $346.998 \mathrm{GHz}$ seen in the image side band. At APEX, when the system is tuned to $\mathrm{ND}_{2} \mathrm{H}$, the $\mathrm{H}^{13} \mathrm{CO}^{+}$line falls $16 \mathrm{MHz}$ outside the $1 \mathrm{GHz}$ wide IF band, but is aliased back into the signal band. The band edge IF filter attenuates the line intensity, therefore the correct intensity scale has been established by comparing the original data with additional measurements taken with APEX on May 22nd, 2006 with the $\mathrm{H}^{13} \mathrm{CO}^{+}$line centered in the signal band. The 2005 data have been multiplied by 2.5 to match the properly tuned 2006 data. We have checked that the spatial distributions of $\mathrm{H}^{13} \mathrm{CO}^{+}(4-3)$ agree reasonably well between both datasets. $\mathrm{H}^{13} \mathrm{CO}^{+}(4-3)$ has a more sharply peaked spatial distribution than $\mathrm{ND}_{2} \mathrm{H}$ (Fig. 2), which is very similar to the $\mathrm{DCO}^{+}(3-2)$ map obtained at the CSO (see right panel of Fig. 2).

\section{Conclusions}

1. We show that the $\mathrm{ND}_{2} \mathrm{H}$ emission is extended in the LDN $1689 \mathrm{~N}$ dense core. The abundance relative to $\mathrm{H}_{2}$, derived from LTE, is $\sim 1.8 \times 10^{-9}$, which makes $\mathrm{ND}_{2} \mathrm{H}$ a remarkably abundant molecule in this dense core. The $\left[\mathrm{ND}_{3}\right] /\left[\mathrm{ND}_{2} \mathrm{H}\right]$ abundance ratio is $0.01-0.02$.
2. The $\mathrm{ND}_{2} \mathrm{H}$ emission is spatially offset from the dust continuum $\mathrm{DCO}^{+}(3-2)$ and $\mathrm{H}^{13} \mathrm{CO}^{+}(4-3)$ emission by $\sim 0.01 \mathrm{pc}$, and appears to be blueshifted relative to the cloud envelope. Both findings are qualitatively consistent with the scenario of the formation of the LDN $1689 \mathrm{~N}$ dense core by the interaction of the blue lobe of the IRAS16293-2422 molecular outflow with the ambient material. Detailed models could further test this scenario.

Acknowledgements. Caltech Submillimeter Observatory is supported by the US National Science Foundation, grant AST 0540882. We thank the MPG and ESO APEX teams for their support, especially P. Bergman and L.- $\AA$. Nyman.

\section{References}

Bergin, E. A., Neufeld, D. A., \& Melnick, G. J. 1999, ApJ, 510, L145

Ceccarelli, C., Vastel, C., Tielens, A. G. G. M., et al. 2000, A\&A, 381, L17

Ceccarelli, C., Loinard, L., Castets, A., et al. A\&A, 372, 998

Coudert, L. H., \& Roueff, E. 2006, A\&A, 449, 855

Dartois, E., Thi, W.-F., Geballe, T. R., et al. 2003, A\&A, 399, 1009

Gerin, M., Pearson, J. C., Roueff, E., Falgarone, E., \& Phillips, T. G. 2001, ApJ, 551, L193

Lesaffre, P., Chièze, J. P., Cabrit, S., \& Pineau des Forêts, G. 2004, A\&A, 427, 147

Lis, D. C., Roueff, E., Gerin, M., et al. 2002a, ApJ, 571, L55

Lis, D. C., Gerin, M., Phillips, T. G., \& Motte, F. 2002b, ApJ, 569, 322

Lis, D. C., Gerin, M., \& Roueff, E. 2006, ApJ, 636, 916

Loinard, L., Castets, A., Ceccarelli, C., Caux, E., \& Tielens, A. G. G. M. 2001, ApJ, 552, L163

Parise, B., Simon T., Caux, E., et al. 2003, A\&A, 410, 897

Ossenkopf, V., \& Henning, T. 1994, A\&A, 291, 943

Pineau des Forêts, G., Roueff, E., \& Flower, D. R. 1989, MNRAS, 240, 167

Roueff, E., Tiné, S., Coudert, L. H., et al. A\&A, 354, L63

Roueff, E., Lis, D. C., van der Tak, F. F. S., Gerin, M., \& Goldsmith, P. F. 2005, A\&A, 438, 585

Stark, R., Sandell, G., Beck, S. C., et al. 2004, ApJ, 608, 341

Tiné, S., Roueff, E., Falgarone, E., Gerin, M., \& Pineau des Forêts, G. 2000, A\&A, 356, 1039 